\title{
Turbulent Taylor-Couette flow over riblets: drag reduction and the effect of bulk fluid rotation
}

\author{
A. J. Greidanus ${ }^{1} \cdot$ R. Delfos ${ }^{1} \cdot$ S. Tokgoz ${ }^{1} \cdot$ J. Westerweel $^{1}$
}

Received: 4 December 2014 / Revised: 3 April 2015 / Accepted: 23 April 2015 / Published online: 12 May 2015

(C) The Author(s) 2015. This article is published with open access at Springerlink.com

\begin{abstract}
A Taylor-Couette facility was used to measure the drag reduction of a riblet surface on the inner cylinder. The drag on the surfaces of the inner and outer cylinders is determined from the measured torque when the cylinders are in exact counter-rotation. The three velocity components in the instantaneous flow field were obtained by tomographic PIV and indicate that the friction coefficients are strongly influenced by the flow regimes and structures. The riblet surface changes the friction at the inner-cylinder wall, which generates an average bulk fluid rotation. A simple model is proposed to distinguish drag changes due to the rotation effect and the riblet effect, as a function of the measured drag change $\Delta \tau_{w} / \tau_{w, 0}$ and shear Reynolds number $R e_{\mathrm{s}}$. An uncorrected maximum drag reduction of $5.3 \%$ was found at $R e_{\mathrm{s}}=4.7 \times 10^{4}$ that corresponds to riblet spacing Reynolds number $s^{+}=14$. For these conditions, the model predicts an azimuthal bulk velocity shift of $1.4 \%$, which is confirmed by PIV measurements. This shift indicates a drag change due to a rotation effect of $-1.9 \%$, resulting in a net maximum drag reduction of $3.4 \%$. The results correspond well with earlier reported results and demonstrate that the Taylor-Couette facility is a suitable and accurate measurement tool to characterize the drag performance of surfaces.
\end{abstract}

A. J. Greidanus

a.j.greidanus@tudelft.nl

1 Laboratory for Aero and Hydrodynamics, Faculty of Mechanical, Maritime and Materials Engineering, Delft University of Technology, Mekelweg 2, 2628 CD Delft, The Netherlands

\section{Introduction}

The reduction of wall friction in turbulent flows has remained an interesting subject for researchers over the last decades. Applications may particular be relevant to industrial devices to reduce the pressure drop in pipe flows, as to decrease fuel consumption and/or to increase transfer speed for transport vehicles. Substantial energy savings for these industries would lead to ecological and economical benefits.

Windtunnels and fluid channels are qualified test facilities to perform turbulent drag reduction studies (Bushnell 1990). However, the forces and the force differences are small and require accurate force measurements in combination with large test surfaces, and these are often the most time-consuming and expensive parts of the research process. An alternative experimental setup for turbulent drag reduction study that is compact, accurate and easy-to-use is a Taylor-Couette system. Several researchers have investigated and reported drag reduction measurements of a flow between two coaxial cylinders, although not all under comparable operational conditions. Experiments with polymers (Kalashnikov 1998), riblets (Hall and Joseph 2000), surfactants (Koeltzsch et al. 2003), highly hydrophobic surfaces (Watanabe et al. 2003) and air bubbles (Berg et al. 2005) are successfully performed and indicate the TaylorCouette facility as a suitable experimental setup to study drag-reducing methods.

The flow in a Taylor-Couette facility presents a flow between two rotating coaxial cylinders. For high Reynolds numbers, the turbulent flow between these presumably infinite long plates in azimuthal direction has a fully developed turbulent shear flow boundary layer. The fluid generates a shear stress on the surfaces that can be determined by measuring the torque on the (inner) cylinder surface. 
Andereck et al. (1986) distinguished and characterized multiple flow regimes and compiled a complex flow pattern diagram. Many parameters influence the different flow states, such as the inner- and outer-cylinder velocity, the gap ratio, the aspect ratio, the end effects and the initial conditions before flow transition between the regimes. Torque scaling research identified the relation between the flow structures and measured torque (Lathrop et al. 1992; Lewis and Swinney 1999; Eckhardt et al. 2007). Ravelet et al. (2010) demonstrated strong dependency of the friction coefficient $C_{\mathrm{f}}$ as a function of the Reynolds number $R e_{\mathrm{S}}$ and the rotation number $R_{\Omega}$, due to the change of turbulent flow structures and their interactions, which was verified by Tokgöz (2014).

In this paper, we discuss our Taylor-Couette facility as an easy-to-use experimental instrument to measure the drag on surfaces. Turbulent drag changes are identified for an adhesive riblet film in the moderate to highly turbulent flow regime (up to $R e_{\mathrm{s}} \approx 1.5 \times 10^{5}$ ). We observe a modification in rotation number for the same mean shear rates due to the use of riblets on the inner cylinder.

In Sect. 2, the experimental and PIV setup are described. We explain the specific parameters and relevant effects for a Taylor-Couette (TC) facility in Sect. 3. Then, in Sect. 4, we discuss the investigation of the TC facility by torque and PIV measurements. We analyze, discuss and validate the results of surface modification with riblets on the inner cylinder and propose a simple model to determine the rotation effect in Sect 5. The main conclusions are summarized in Sect. 6.

\section{Taylor-Couette facility and PIV setup}

\subsection{Taylor-Couette facility}

The TC facility in the present study was previously used in the investigation of Ravelet et al. (2010), Tokgoz et al. (2012) and Tokgöz (2014). It consists of two coaxial acrylic glass cylinders that both can rotate independently. The inner cylinder is sealed by PVC end-plates at the top and bottom. The radius of the inner cylinder is $r_{\mathrm{i}}=110 \mathrm{~mm}$ and total length $L_{\mathrm{i}}=216 \mathrm{~mm}$. The outer cylinder has a radius $r_{\mathrm{O}}=120 \mathrm{~mm}$ and a length $L_{\mathrm{O}}=220 \mathrm{~mm}$ (Fig. 1). The inner cylinder is assembled within the outer cylinder with high precision; the shaft is secured by two ball bearings at the top, while the bottom remains centered and stabilized by a polymer ball bearing in the bottom end-plate of the inner cylinder. The radial gap between the cylinders is $d=r_{\mathrm{O}}-r_{\mathrm{i}}=10.0 \mathrm{~mm}$, and hence the gap ratio is $\eta=r_{\mathrm{i}} / r_{\mathrm{o}}=0.917$ and axial aspect ratio is $\Gamma=L_{i} / d=21.6$.

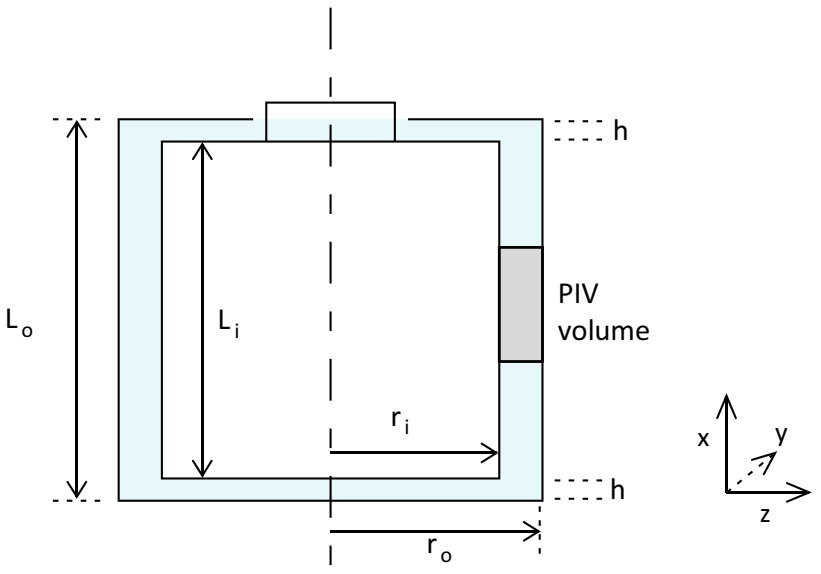

Fig. 1 Sketch of the Taylor-Couette facility in the radial-axial plane. The coordinate system in the measurement volume is given by $x$ for axial, $y$ for azimuthal and $z$ for radial direction. The dimensions are not to scale

The system is closed by end-plates attached to the outer cylinder and are rotating with the outer cylinder. The gaps between the top and bottom end-plates of the inner and outer cylinders are around $h=2 \mathrm{~mm}$ and are called the von Kármán-gaps. The fluid motions in these horizontal gaps (i.e. von Kármán flow) create an additional fluid friction on the cylinders during rotation (Daily and Nece 1960; Hudson and Eibeck 1991; Kilic and Owen 2002). The mechanical friction of the system bearings can be neglected compared to the total fluid friction in the system, as was verified with an empty system. The total torque $\left(M_{\text {Tot }}\right)$ on the inner cylinder is recorded with a co-rotating torque meter (HBM T20WN/2Nm, abs. precision $\pm 0.01 \mathrm{Nm}$ ) that is assembled in the shaft between the driving motor (Maxon, $250 \mathrm{~W}$ ) and inner cylinder. The outer cylinder is driven by an identical external motor via a driving belt.

The shear stress $\tau$ is among others influenced by the temperature-dependent fluid viscosity. During measurements, we observe the temperature change in the working fluid closely as we are not able to control the fluid temperature. When the cylinders are at rest, the fluid temperature $T_{\mathrm{f}}$ is manually measured via an opening in the top lid by a thermocouple (RS, type $\mathrm{K}$ ) connected to a digital thermometer (RS1319A). When the cylinders are rotating, it is not possible to measure the fluid temperature directly since the system is completely closed. Instead, the outside wall temperature $T_{\text {out }}(t)$ of the outer cylinder is recorded in time by an infrared-thermometer (Calex PyroPen). The fluid temperature $T_{\mathrm{f}}(t)$ is indirectly determined via heat transfer calculations, based on the material and fluid properties of the setup. At the end of a measurement, the temperature value is verified by manual temperature measurement as described before and agrees very well $\pm 0.2{ }^{\circ} \mathrm{C}$. The 
Table 1 Glycerol/water fluid properties: mass fraction glycerol, fluid viscosity at $20-25^{\circ} \mathrm{C}$, heat capacity coefficient and viscous sensitivity of temperature change $\left(20-25^{\circ} \mathrm{C}\right)$.

\begin{tabular}{lllll}
\hline Mixture & $C_{m}(\mathrm{wt} \%)$ & $\begin{array}{l}10^{6} \times v \\
\left(\mathrm{~m}^{2} / \mathrm{s}\right)\end{array}$ & $\begin{array}{l}c_{p} \\
(\mathrm{~kJ} /(\mathrm{kg} \mathrm{K}))\end{array}$ & $\begin{array}{l}\partial\left(\mathrm{v} / \nu_{0}\right) / \\
\partial T(\% / \mathrm{K})\end{array}$ \\
\hline 5 & 85.9 & 100 & 2.68 & -6.6 \\
4 & 62.1 & 10.8 & 3.09 & -4.3 \\
3 & 52.6 & 6.09 & 3.26 & -3.7 \\
2 & 38.9 & 3.19 & 3.50 & -3.2 \\
1 & 17.1 & 1.53 & 3.88 & -2.6 \\
0 & 0 & 1.01 & 4.18 & -2.4 \\
\hline
\end{tabular}

determined fluid temperatures $T_{\mathrm{f}}(t)$ are used to estimate the correct value of the working fluid viscosity during torque measurements. The working fluids in the present study are various glycerol-water mixtures, with a kinematic viscosity in the range of $1.0 \times 10^{-6}<v<100 \times 10^{-6} \mathrm{~m}^{2} / \mathrm{s}$ at $20{ }^{\circ} \mathrm{C}$ (Table 1).

Experiment control and data acquisition (DAQ) are accomplished with a desktop computer. The two motors are controlled by a software program (LabVIEW, National Instruments Corp.) that regulates the desired angular velocities of the inner and outer cylinders. The torque meter is connected via a DAQ block (NI PCI-6035E) to a 12-bit DAQ board (NI BNC-2110) that records the torque and rotation rate signal of the inner cylinder at a sampling rate of $2 \mathrm{kHz}$ for $120 \mathrm{~s}$. Simultaneously, the outside wall temperature signal $T_{\text {out }}(t)$ of the IR-thermometer is recorded by a manufacturer supplied software (Calexsoft $1.05)$.

\subsection{PIV setup}

The three velocity components in the instantaneous flow field can be measured by tomographic particle image velocimetry (tomo-PIV, Elsinga et al. 2006). The application of the tomo-PIV to Taylor-Couette is shown in Fig. 2 and is described in more detail by Tokgöz (2014). Particle images were recorded with four cameras (Imager Pro $X 4 \mathrm{M})$ in double frame mode with a resolution of $2000 \times 2000$ pixels. A double-pulsed Nd:YAG laser $(\mathrm{New}$ Wave Solo-III) was used to illuminate the measurement volume between the two cylinders with a volume size of roughly $40 \times 20 \times 10 \mathrm{~mm}^{3}$ in axial, azimuthal and radial directions. The measurement volume is located at midheight of the rotational axis to minimize the possible end effects of the Taylor-Couette facility on the measurements (Fig. 1).

Fluorescent particles (Fluostar) with a mean diameter of $15 \mu \mathrm{m}$ were used as tracer particles. The seeding concentration was around 0.025 particles per pixel. This is considered

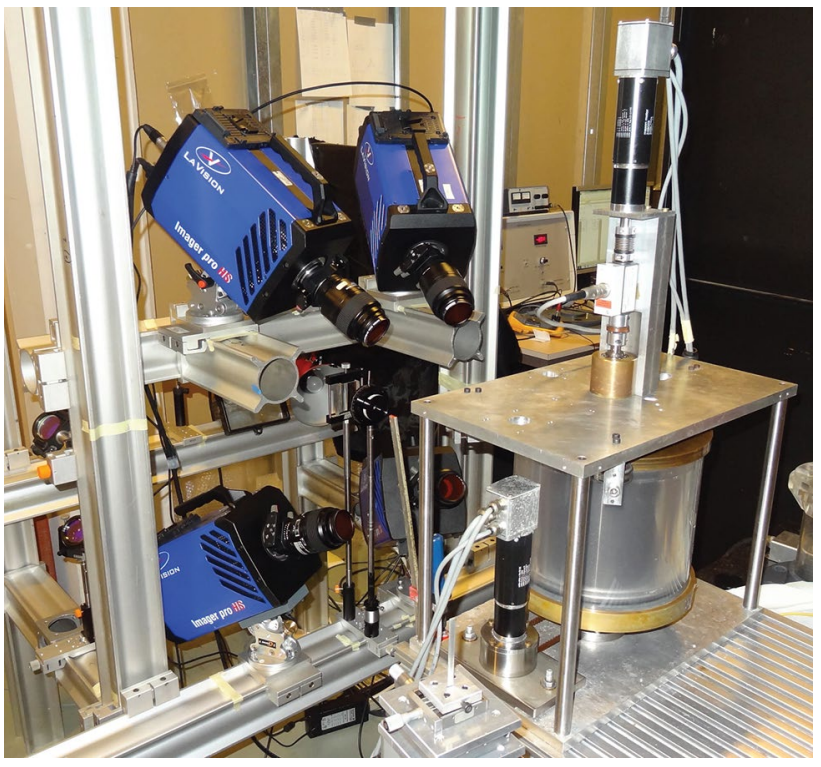

Fig. 2 Experimental setup with PIV cameras. The cameras on the photo differs from the cameras mentioned in the text

to be an optimal seeding concentration to achieve a high spatial resolution and rule out speckle effects in the recorded images (Tokgoz et al. 2012). The sharp contrast between the intensity inside and outside the volume gap in radial direction indicates a high quality of the tomographic reconstruction.

For the calibration procedure a stainless steel flat plate of $1 \mathrm{~mm}$ thick was used as a calibration target with $0.4 \mathrm{~mm}$ circular holes in repetition at every $2.5 \mathrm{~mm}$ in the vertical and horizontal directions. The calibration target was stably attached to a translating and rotating microtraverse, capable of positioning the target with high precision. Calibration images were recorded in three selected planes in the radial direction, with $2.5 \mathrm{~mm}$ distance in between.

The quality of the recorded images was improved by image processing using commercial software (Davis by LaVision). The signal-to-noise ratio was increased by first subtracting a sliding minimum intensity of $25 \times 25$ pixels from all images followed by a $3 \times 3$-pixel Gaussian smoothing filter. Tomographic reconstruction was performed with the MART algorithm (Elsinga et al. 2006). A multipass correlation was used with a final interrogation window of $40 \times 40 \times 40$ voxels with a $75 \%$ overlap. The universal outlier detection method (Westerweel and Scarano 2005) removed spurious vectors, and the resulting gaps were filled by linear interpolation.

\subsection{Riblets}

Longitudinal ribbed surfaces, called riblets, are able to reduce the skin friction in a turbulent boundary layer in 
comparison with smooth surfaces (Bechert et al. 1997). The reduction depends on the dimensions and geometry of the riblets in relation to the local flow. The riblet spacing Reynolds number, or dimensionless riblet spacing, is traditionally defined by the parameter $s^{+}=s u_{\tau} / v$, where $s$ is the riblet spacing, $u_{\tau}\left(=\sqrt{\tau_{w, 0} / \rho}\right)$ is the friction velocity. The drag reduction is expressed in the change in shear stress compared to a smooth surface $\left(\Delta \tau_{w} / \tau_{w, 0}\right)$. Several types of riblet geometries are known, which can typically reduce the skin friction up to 5-10\%.

In this study, we use a 3M Scotchcal High Performance Film with longitudinal grooves as riblet surface, which has been used in previous research (van der Hoeven 2000). A picture taken with scanning electron microscopy (SEM) shows a triangular cross-sectional geometry of the riblets with a spacing $s=120 \mu \mathrm{m}$ and height $h= \pm 110 \mu \mathrm{m}$ (Fig. 3). The rib tips have a noticeable round/flat area, which can have a negative effect on the drag-reducing performance of riblets (Bechert et al. 1997).

The film is adhered to the surface with high precision to avoid air pockets and misalignment of the grooves, which should be parallel to the azimuthal flow. A small axialaligned gap of roughly $0.3 \mathrm{~mm}$ is observed between the edges of the film and makes an irregularity in the cylinder surface. However, the film gap is very small and the torque contribution of the present gap is considered to be marginal. The total film thickness measured from the bottom of the sheet to the tops of the riblet is $190 \mu \mathrm{m}$ and the adhered riblet film results in a small change in the inner-cylinder radius and the radial gap, which is taken into account for the experimental conditions.

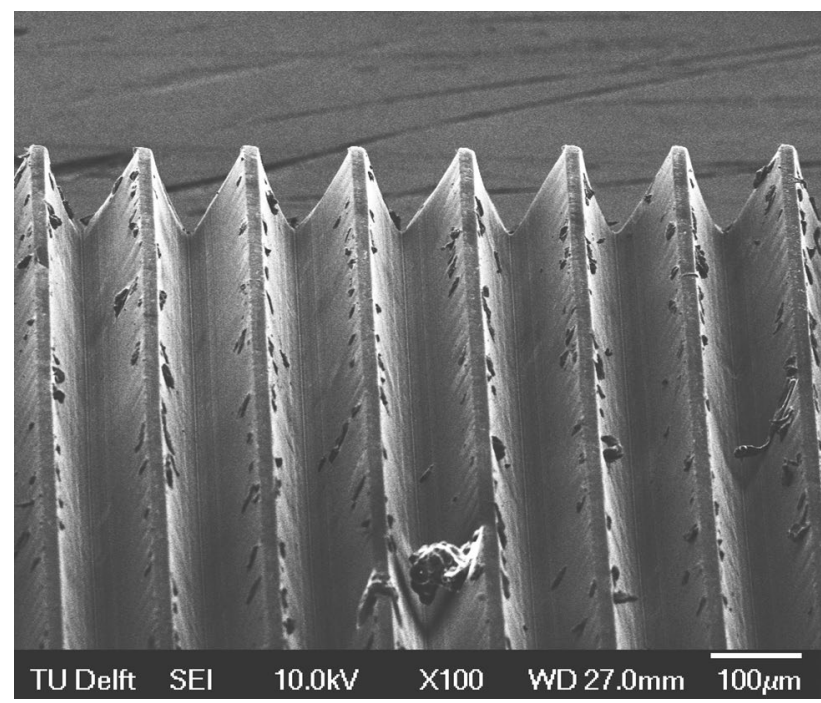

Fig. 3 SEM picture of sawtooth riblet surface, view-angle 70 degrees. Riblet spacing $120 \mu \mathrm{m}$, riblet height $\pm 110 \mu \mathrm{m}$. Rib-tip to spacing ratio $t / s \simeq 0$.1. Photo taken by C. Kwakernaak ( $3 \mathrm{mE}-\mathrm{TU}$ Delft)

\section{Specific parameters and relevant Taylor- Couette effects}

In this section, we describe the control parameters that applies to rotating shear flows in general. The flow characteristics are determined by the angular velocities and radii of the inner and outer cylinders, the gap between the cylinders and the fluid viscosity. The use of finite length cylinders induces end effects on the torque measurements and creates flow instabilities.

\subsection{Reynolds and rotation numbers}

The shear Reynolds number of Taylor-Couette flows is comparable with the Reynolds number of plane Couette flows in a rotating frame. Dubrulle et al. (2005) introduced a new set of control parameters based on dynamical rather than the traditional geometrical considerations. Traditionally based on the gap width $d=r_{\mathrm{o}}-r_{\mathrm{i}}$ between the two cylinders, the inner- and outer-cylinder Reynolds numbers are, respectively, defined as $R e_{\mathrm{i}}=\left(r_{\mathrm{i}} \omega_{\mathrm{i}} d / \nu\right)$ and $R e_{\mathrm{o}}=\left(r_{\mathrm{o}} \omega_{\mathrm{o}} d / \nu\right)$, where $\omega_{\mathrm{i}}$ and $\omega_{\mathrm{o}}$ are the angular velocities and $v$ is the kinematic viscosity of the fluid. The sign of the Reynolds number indicates the rotation direction of the cylinders. The parameters are combined to introduce the dynamical control parameters: the shear Reynolds number $R e_{\mathrm{S}}$, rotation number $R_{\Omega}$ and curvature number $R_{\mathrm{C}}$ are defined as:

$R e_{\mathrm{S}}=\frac{2}{1+\eta}\left|\eta R e_{\mathrm{o}}-R e_{\mathrm{i}}\right|$

$R_{\Omega}=(1-\eta) \frac{R e_{\mathrm{i}}+R e_{\mathrm{o}}}{\eta R e_{o}-R e_{\mathrm{i}}}$,

$R_{\mathrm{C}}=\frac{1-\eta}{\eta^{1 / 2}}$.

The shear Reynolds number $R e_{\mathrm{S}}$ is based on the shear rate between the inner and outer cylinders. The rotation number $R_{\Omega}$ indicates the net system rotation and represents the influence of the mean rotation on the shear. The sign of the rotation number defines the case of cyclonic $\left(R_{\Omega}>0\right)$ or anti-cyclonic $\left(R_{\Omega}<0\right)$ flows. For inner- and outer-cylinder rotation only, the rotation numbers are $R_{\Omega, \mathrm{i}}=\eta-1$ and $R_{\Omega, 0}=(1-\eta) / \eta$ respectively. In case of exact counterrotation $\left(r_{\mathrm{i}} \omega_{\mathrm{i}}=-r_{\mathrm{o}} \omega_{\mathrm{o}}\right)$, the rotation number $R_{\Omega}$ is zero and indicates that the mean bulk velocity $\bar{U}_{b}$ is zero. The curvature number, dependent on the gap ratio $\eta$, indicates the effect of the cylinder radii on the stability of circumferential flows. The cylinder gap $d$ in our setup is considered to be narrow, with a gap ratio $\eta=r_{\mathrm{i}} / r_{\mathrm{o}} \simeq 0.917$, i.e. close to unity. The curvature number in our experiments is therefore $R_{\mathrm{C}} \simeq 0.087$. 


\subsection{Torque contribution of the Taylor-Couette and von Kármán flows}

The fluid inside the experimental setup is distributed into two main regions: the gap between the inner- and outercylinder wall (Taylor-Couette gap) and the gap between the end-plates of the cylinders (von Kármán gap). The fluid motions in these regions are very different and demands an other type of methodology to obtain the shear forces working on the inner cylinder.

The torque on the cylinder walls $\left(M_{\mathrm{TC}}\right)$ is defined by the wall shear stress $\tau_{w}$ multiplied by the surface area of the cylinder $A$ and lever arm $r$ :

$M_{\mathrm{TC}}=2 \pi L_{i} r_{i}^{2} \tau_{w, i}=2 \pi L_{i} r_{o}^{2} \tau_{w, o}$.

The skin friction coefficient $C_{\mathrm{f}}$ is a dimensionless parameter, which is used to analyze drag changes when the surface of the inner cylinder is modified. The friction coefficient $C_{\mathrm{f}}$ is the ratio of wall shear stress $\tau$ and the dynamic pressure $\frac{1}{2} \rho U_{\mathrm{sh}}^{2}$ (Ravelet et al. 2010):

$C_{\mathrm{f}}=\frac{M_{\mathrm{TC}}}{\pi \rho r_{i}^{2} L_{i} U_{s h}^{2}}$.

In (5) the shear velocity $U_{\mathrm{sh}}$ is proportional to $R e_{\mathrm{s}} \mathrm{v} / d$. Equation (5) represents the friction coefficient for laminar and turbulent flow conditions. The analytical solution of the friction coefficient for laminar flow, with shear velocity $U_{\mathrm{sh}}=\omega_{o} r_{o}-\omega_{i} r_{i}$, is given by:

$C_{\mathrm{f}}=\frac{4 v\left(\omega_{o}-\omega_{i}\right)}{\left(1-\eta^{2}\right)\left(\omega_{o} r_{o}-\omega_{i} r_{i}\right)^{2}}$.

The fluid motions in the horizontal von Kármán gap (vK-gap) create an additional fluid friction on the cylinders. The shear flow between the end-plates applies a torque on the inner-cylinder end-plates with radius $r_{i}$, with the outer-cylinder endplates rotating at a gap height $h$ in between. The torque on the inner cylinder under laminar flow conditions realized by the fluid motion in the vK-gap can be expressed by:

$M_{\mathrm{vK}}=\frac{\pi \rho v\left(\omega_{o}-\omega_{i}\right) r_{i}^{4}}{2 h}$.

Combining (5-7) gives the ratio between the torque contribution of the two vK-gaps and the TC-gap for laminar flows, considering the gap height of the bottom and top vKgap $(h)$ to be similar:

$\frac{2 M_{\mathrm{vK}}}{M_{\mathrm{TC}}}=\frac{r_{i}^{2}\left(1-\eta^{2}\right)}{4 h L_{i}}$.

Equation (8) shows that the measured torque $M_{\text {tot }}$ becomes more dependent of the fluid friction in the TC-gap when the vK-gap height $h \rightarrow \infty$ or when $L_{i} \rightarrow \infty$. Under laminar flow conditions, the described experimental setup has a torque ratio of $M_{\mathrm{vK}} / M_{\mathrm{TC}} \simeq 1$.

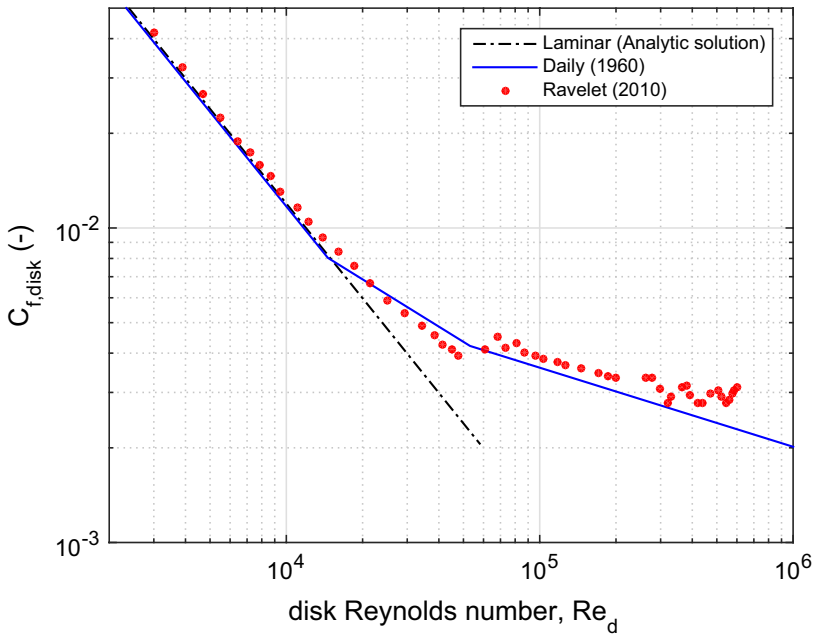

Fig. 4 The von Kármán disk friction coefficient $C_{\mathrm{f} \text {,disk }}$ as a function of the disk Reynolds number $R e_{\mathrm{S}}=|\Delta \omega| r_{i}^{2} / \nu$ for an axial spacing/ disk radius ratio $h / r_{i}=0.02$

For turbulent flow conditions, the torque contribution of the von Kármán gaps is assumed to be independent of $R_{\Omega}$ and is estimated to be equal to half of the measured torque at $R_{\Omega}=0.091$, as suggested by Tokgöz (2014). This assumption is compared to the results of Daily and Nece (1960). They studied the flow regimes in the axial gap between a rotation disk enclosed within a right-cylindrical chamber, and it was verified that the disk friction coefficient $C_{\mathrm{f} \text {,disk }}$ is a function of the disk Reynolds number $R e_{\mathrm{d}}=|\Delta \omega| r_{i}^{2} / \nu$ and the axial spacing/disk radius ratio $h / r_{i}$. Figure 4 shows the relation between $C_{\mathrm{f}, \text { disk }}$ and $R e_{\mathrm{d}}$ for an axial spacing/disk radius ratio $h / r_{i}=0.02$.

The measured torque of only outer-cylinder rotation $\left(R_{\Omega}=0.091\right)$ of Ravelet et al. (2010) is used to determine the disk friction coefficient $C_{\mathrm{f} \text {,disk }}$ of the end-plate in the von Kármán gap for the current setup. Figure 4 indicates that the assumption fits reasonably well to the results found by Daily and Nece (1960).

The torque of the fluid motions in the von Kármán gap $M_{\mathrm{vK}}$ for the current setup is estimated by:

$M_{\mathrm{vK}}=C_{\mathrm{f}, \mathrm{disk}} \frac{1}{5} \pi \rho\left(\omega_{o}-\omega_{i}\right)^{2} r_{i}^{5}$.

\subsection{Azimuthal velocity profile}

Taylor derived an exact solution for the laminar azimuthal velocity profile of the flow between two infinitely long coaxial cylinders (Taylor 1923). In practice, the cylinders have a finite length and the azimuthal flow experiences disturbances from the fluid motions in the horizontal vK-gaps that penetrates into the vertical TC-gap, causing a secondary flow. This leads to flow instabilities at lower Reynolds number than the theoretical predicted critical Reynolds 
number for flow transitions (Esser and Grossmann 1996). It is assumed that the end effects will not influence the bulk profile in the turbulent regime as a secondary flow is much weaker than the turbulent fluid motions.

The turbulent velocity profile of the azimuthal flow is more complex. Dong (2008) performed 3D direct numerical simulations of the turbulent flow between exact counter-rotating cylinders $\left(R_{\Omega}=0\right)$ with a gap ratio $\eta=0.5$ at moderate Reynolds numbers that correspond to featureless turbulence and unexplored turbulent regions in the flow pattern diagram of Andereck et al. (1986). They report an asymmetrical azimuthal velocity profile at the cylinder walls and a core flow which has near-zero azimuthal velocities at higher Reynolds numbers. This region of nearzero azimuthal velocities expands as the Reynolds number increases. The velocity profile approaches a linear line with slope $\alpha$ in the core of the flow $\left(U_{\theta, b u l k}(r)=\alpha\left(r-r_{i}\right) / d\right)$ and the location of the surface with zero azimuthal velocities shifts outwards when the Reynolds number were increased, with a limiting value of $\left(r^{*}-r_{i}\right) / d \sim 0.55$.

\section{Investigation of the experimental conditions}

\subsection{Experimental considerations}

In order to make a proper drag performance comparison, it is preferable to measure the riblet surfaces under similar flow conditions as for boundary layer or fluid channel experiments. Global rotation, which is the rotation of the frame of reference in which the shear flow occurs, can strongly influence the turbulent structures (Bokhoven et al. 2009). With regard to a Taylor-Couette setup, global rotation can enhance or suppress turbulence for only inner- or outer-cylinder rotation, respectively. Therefore, the measurements are performed under exact counter-rotation, with a rotation number $R_{\Omega}=0$.

Frictional heating causes thermal effects on the estimation of the correct fluid viscosity, as was mentioned by Hall (Hall and Joseph 2000). Nevertheless, a flow under exact counter-rotating conditions is indicated as highly turbulent and therefore well mixed, resulting in a constant fluid temperature over the radial gap. The fluid temperature $\pm 0.2{ }^{\circ} \mathrm{C}$ is indirectly determined via heat transfer calculations and deviates maximum $\pm 0.2{ }^{\circ} \mathrm{C}$ from the actual fluid temperature when checked manually. For common turbulent flows, the friction coefficient $C_{\mathrm{f}}$ scales with $R e^{-1 / 4}$ and results in a maximum error of the friction coefficient of $0.1-0.3 \%$ due to the temperature deviation.

The drag-reducing material, in this case the riblet film, is only applied on the inner-cylinder surface as it is much easier, faster and more accurate to apply. The outer-cylinder surface remains unaltered, as the riblet film is also non-transparent and inhibits PIV-measurements. A consequence is that the inner- and outer-cylinder surfaces have different skin-friction coefficients and modify the condition for the rotation number $R_{\Omega}$ of exact counter-rotation. This is discussed in Sect. 5.1.

\subsection{Torque measurements}

For all Reynolds numbers, the torque contribution of the TC-gap is determined by the time-averaged values of the measured torque minus the torque values of the vK-gap at $R_{\Omega}=0.091$ and are used to determine the friction coefficients $C_{\mathrm{f}}$. Figure 5 shows the friction coefficient $C_{\mathrm{f}}$ of an untreated, smooth cylinder surface as a function of the shear Reynolds number $R e_{\mathrm{S}}$ for a rotation number $R_{\Omega}=0$. The current data are identical to the data of Ravelet et al. (2010) and are similar to the results of planar-Couette flow (Aydin and Leutheusser 1991). The friction coefficients in Fig. 5 are used as a reference to identify drag changes due to surface modification of the inner cylinder. The results of the friction coefficient $C_{f}$ are reproducible within $0.6 \%$ for all working fluids. At low Reynolds numbers $\left(R e_{\mathrm{S}}<400\right)$, the curve is identical to the theoretically predicted curve of $C_{\mathrm{f}}(6)$ and validates the torque contribution of $50 \%$ of the TC-gap. The measured torque for laminar flow is linear with the relative angular velocity and scales as $C_{\mathrm{f}} \sim 1 / R e$. The first flow transition already occurs at $R e_{\mathrm{s}} \simeq 400$, which

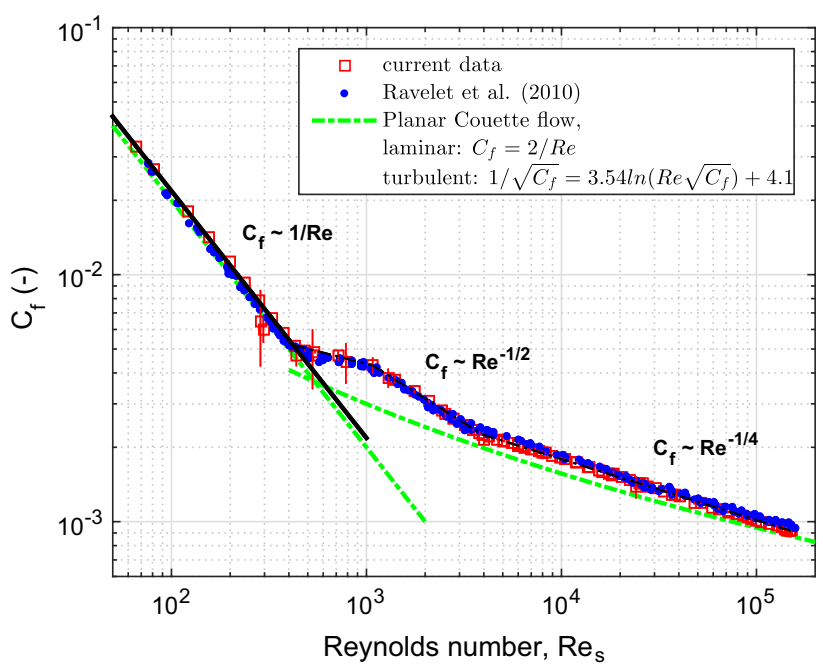

Fig. 5 Friction coefficient $C_{\mathrm{f}}$ as a function of shear Reynolds number $R e_{\mathrm{s}}$ under exact counter-rotation $\left(R_{\Omega}=0\right)$. Error bars represent the estimated $95 \%$ confidence interval. The solid black line represents the analytical solution for laminar flow and scales with $C_{\mathrm{f}} \sim 1 / R e$. The Taylor and fully turbulent regime are scaled with $C_{\mathrm{f}} \sim R e^{-1 / 2}$ and $C_{\mathrm{f}} \sim R e^{-1 / 4}$, respectively. Current data compared to the data of Ravelet et al. (2010) and plane-Couette flow. Laminar plane-Couette flow, $C_{\mathrm{f}}=2 / R e$. Turbulent plane-Couette flow, $1 / \sqrt{C_{\mathrm{f}}}=3.54 \ln \left(\operatorname{Re} \sqrt{C_{\mathrm{f}}}\right)+4.1$ (Aydin and Leutheusser 1991) 
is lower than the theoretical value of $R e_{\mathrm{s}}=515$ (Esser and Grossmann 1996). We believe that the finite aspect ratio induces secondary flow that creates an earlier state of instability. A transitional regime arises between $R e_{\mathrm{s}} \sim 400$ and 1000 , and above $R e_{\mathrm{S}}>1000$ the friction coefficient scales with $C_{\mathrm{f}} \sim R e^{-1 / 2}$. A second flow transition occurs around $R e_{\mathrm{S}} \sim 4000$, and for higher Reynolds numbers the curve scales with $C_{\mathrm{f}} \sim R e^{-1 / 4}$. The flow is considered to be fully turbulent under these conditions.

\subsection{Velocity profile: PIV measurements}

The three velocity components of instantaneous flow fields are obtained by tomo-PIV measurements. The reliability of the tomo-PIV method of this experimental setup has been evaluated and described by Tokgoz et al. (2012). In the past, riblet surfaces have been successfully applied to reduce skin friction in turbulent boundary layers and therefore turbulent velocity components in the TC-gap are highly interesting to investigate. We have used PIV data of turbulent flow at Reynolds numbers $R e_{\mathrm{S}}=3800$ to $4.7 \times 10^{4}$ under exact counter-rotating conditions $\left(R_{\Omega}=0\right)$, which were presented previously by Tokgoz et al. (2012). The averaged velocities of the azimuthal flow as a function of the radial position are given in Fig. 6. The velocities are obtained by averaging over 1000 instantaneous 3D velocity fields and in axial direction. Data points close to the walls are excluded from the measured domain as they are not reliable due to optical distortions. The minimum and maximum estimated $95 \%$ confidence intervals of the azimuthal velocity data are listed in the table of Fig. 6. The minimum and maximum errors are related to the data points in the core flow or close to the walls, respectively. The averaged azimuthal velocity profiles suggest high velocity gradients near both walls and low velocity gradients in the middle of the gap (core flow). The velocity gradient at the wall increases by increasing the Reynolds number $R e_{\mathrm{s}}$, which indicates a higher level of turbulence. The zero-crossing where the averaged azimuthal velocity changes from negative to positive is around $\left(r^{*}-r_{\mathrm{i}}\right) / d \sim 0.52-0.56$ for all cases. The core of the flow has very low azimuthal velocities, and when the Reynolds number increases, the azimuthal velocity profile of the core flow approaches a straight line with slope $\alpha \approx 0.25$, indicated by the dashed line in Fig. 6. The averaged axial and radial velocity profiles are within a maximum value of $0.6 \%$ of the outer-cylinder wall velocity. The instantaneous PIV data show axial and radial velocity fluctuations of $\pm 10-20 \%$ of the outercylinder wall velocity.

Figure 7 presents the azimuthal vorticity and the velocity vectors of the time-averaged flow in the radial-axial plane of the gap. The presence of large-scale structures is clearly visible at $R e_{\mathrm{S}}=3800$, which are counter-rotating

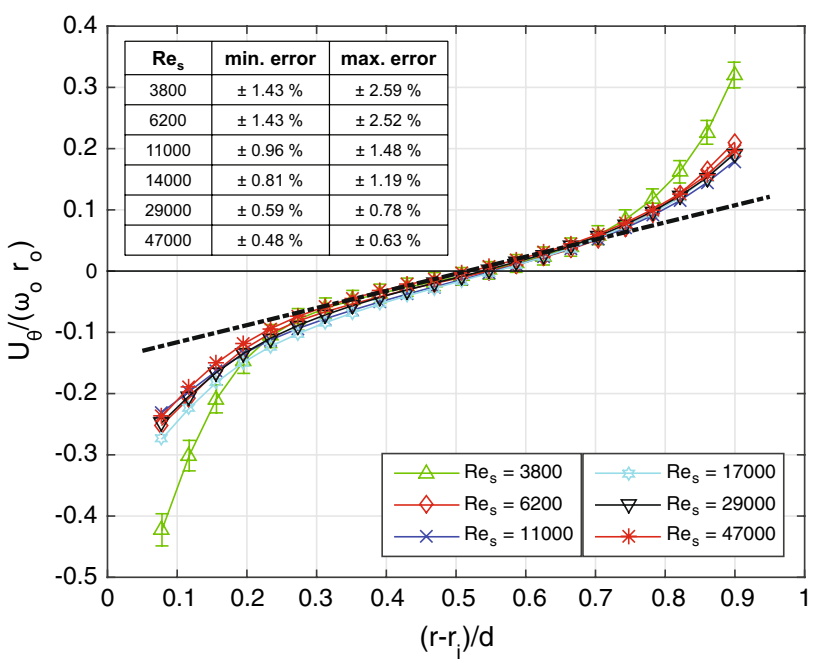

Fig. 6 Averaged azimuthal velocities under exact counter-rotation $\left(R_{\Omega}=0\right)$ as a function of the radial position. The velocities are normalized to the azimuthal velocity of the outer cylinder $\left(\omega_{0} r_{0}\right)$. The minimum and maximum estimated $95 \%$ confidence intervals of the azimuthal velocity data are presented in the table. Errorbars are only shown for $R e_{\mathrm{S}}=3800$. The dashed line represents the azimuthal core flow velocity for turbulent flows, with slope $\alpha \approx 0.25$

Taylor-vortices with a diameter comparable to that of the TC-gap width. The structures are much weaker at $R e_{\mathrm{S}}=6200$ and also small-scale structures arise. The large vortex structures have completely disappeared for fully turbulent flows. The vorticity plots indicate a least limited Reynolds number at which the flow in the Taylor-Couette facility has typical turbulent flow characteristics comparable to channel or pipe flows. The flow at $R e_{\mathrm{s}}=3800$ and $R e_{\mathrm{S}}=6200$ have visible Taylor-vortices and therefore the riblet results under these conditions are considered to be an artifact of the experimental setup rather than the drag characteristics of riblets.

In conclusion, Figs. 5, 6, 7 show that the friction coefficient is strongly related to the flow regime (laminar, Taylor-vortices and fully turbulent flow) with different scaling $\left(C_{\mathrm{f}} \sim R e^{-1}, R e^{-1 / 2}\right.$ and $R e^{-1 / 4}$, respectively).

Furthermore, the derived riblet data will be limited to $R e_{\mathrm{S}}>10^{4}$ in order to make a suitable comparison to data obtained by other researchers.

\section{Results}

Figure 8 presents the determined friction coefficients $C_{\mathrm{f}}$ against shear Reynolds number $R e_{\mathrm{s}}$ for smooth and riblet inner-cylinder surfaces under exact counter-rotating conditions $\left(R_{\Omega}=0\right)$. For low Reynolds numbers up to $R e_{\mathrm{s}}=4.0 \times 10^{3}$, the friction coefficients are slightly higher 

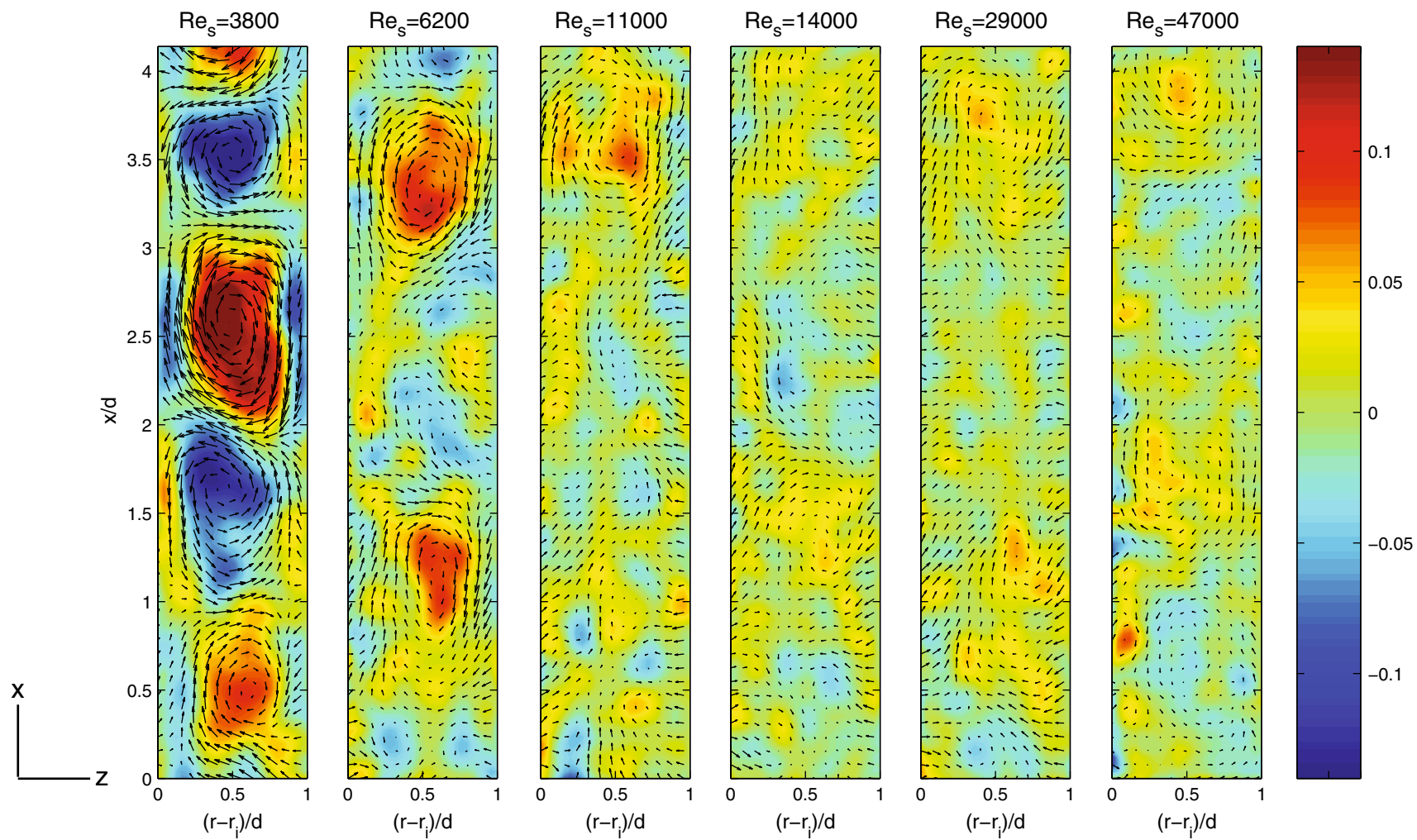

Fig. 7 Vorticity plot color indicates the normalized strength of the out-of-plane vorticity $(\mathrm{d} \bar{U} / \mathrm{d} z-\mathrm{d} \bar{W} / \mathrm{d} x) /\left(\omega_{\mathrm{o}} r_{\mathrm{o}} / d\right)$, arrows indicate the radial and axial velocities. The vorticity and velocity in each plot are normalized to outer-cylinder wall velocity $\omega_{\mathrm{o}} r_{\mathrm{o}}$ and radial gap length $d$

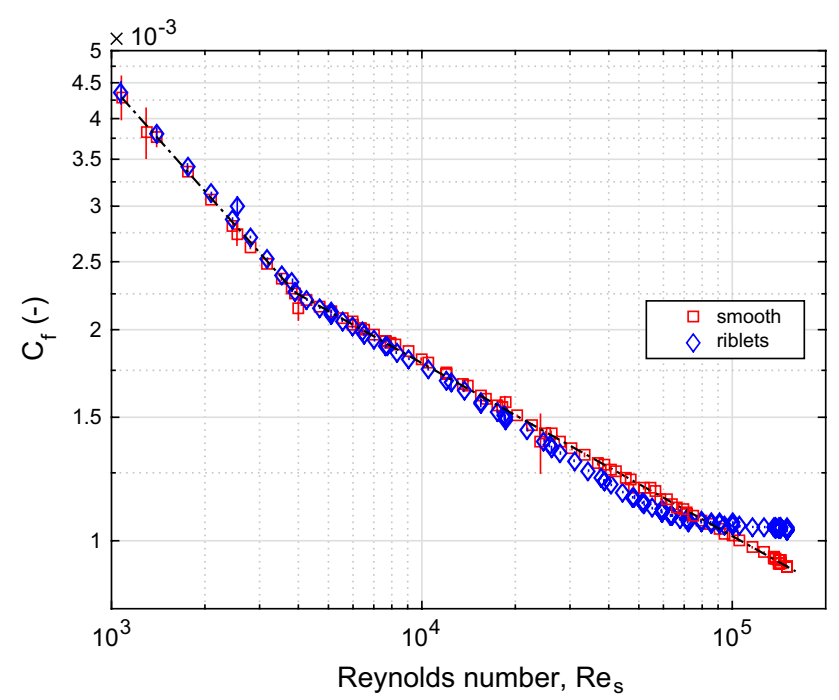

Fig. 8 Friction coefficient $C_{\mathrm{f}}$ vs shear Reynolds number $R e_{\mathrm{s}}$ under exact counter-rotation $\left(R_{\Omega}=0\right)$ of a smooth (red circle) and a riblet (blue diamond) inner-cylinder surface, for $R e_{\mathrm{s}}=1000$ to $15 \times 10^{4}$

compared to a smooth surface. For higher Reynolds numbers, the friction values are initially lower, while for Reynolds numbers $R e_{\mathrm{s}}>8.5 \times 10^{4}$ the values are much higher compared to a smooth surface. An alternative figure to indicate the drag change is given in Fig. 9. The change in drag at the inner-cylinder surface is determined by:

$\frac{\Delta \tau_{w}}{\tau_{w, 0}}=\frac{\tau_{w, r i b}-\tau_{w, 0}}{\tau_{w, 0}}=\frac{C_{f, r i b}-C_{f, 0}}{C_{f, 0}}$.

A drag reduction is observed for a Reynolds number interval of $R e_{\mathrm{S}}=4.0 \times 10^{3}$ to $8.5 \times 10^{4}$, with a maximum apparent drag reduction of $5.3 \%$ around $R e_{\mathrm{S}}=4.7 \times 10^{4}$. Drag increases in the Reynolds regime with Taylorvortices (Fig. 9, inset), as for Reynolds numbers above $R e_{\mathrm{s}}=8.5 \times 10^{4}$. A maximum drag increase of $14 \%$ is noticed for a Reynolds number $R e_{\mathrm{s}}=15 \times 10^{4}$. For further evaluation, the riblet data will be limited to $R e_{\mathrm{S}}>10^{4}$ as is stated in Sect. 4.3.

\subsection{Rotation effect}

The fluid motions in the TC-gap are driven by the surfaces of the inner and outer cylinders and generate an angular momentum balance, or torque balance $\left(M_{\mathrm{tc}, \mathrm{i}}=M_{\mathrm{tc}, \mathrm{o}}\right)$. Equation (4) indicates that the shear stress at the innercylinder wall is larger than at the outer-cylinder wall. The shear stress at the inner-cylinder surface is given in (11), with $\bar{U}_{\mathrm{b}}$ as the averaged azimuthal bulk/core velocity. 


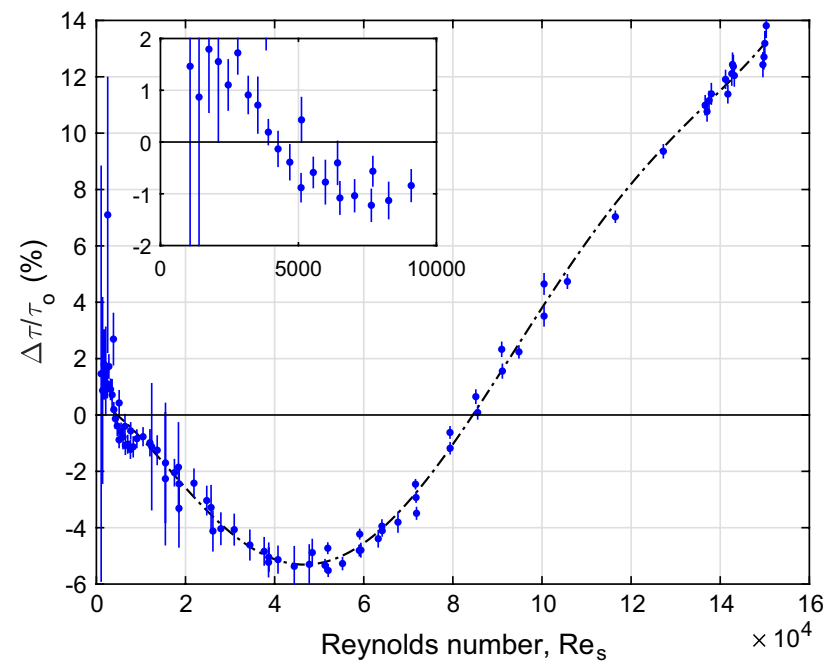

Fig. 9 Measured drag change under exact counter-rotation $\left(R_{\Omega}=0\right)$ . The dashed line represents the polynomial fit. Inset Zoom-in view around flow transition: Taylor-vortex to turbulent regime. Errorbars $95 \%$ confidence interval

$$
\begin{aligned}
\tau_{w} & =\frac{1}{2} \rho C_{f i}\left(U_{\text {in }}-\bar{U}_{\mathrm{b}}\right)^{2} \\
& =\frac{1}{2} \rho C_{f o}\left(U_{\text {out }}-\bar{U}_{\mathrm{b}}\right)^{2}\left(\frac{r_{\mathrm{o}}}{r_{\mathrm{i}}}\right)^{2} .
\end{aligned}
$$

The friction measurements are executed under exact counter-rotation $\left(U_{\text {out }}=-U_{\text {in }}\right)$. The azimuthal velocity profile of a smooth inner- and outer-cylinder surface at Reynolds number $\operatorname{Re}_{\mathrm{s}}=4.7 \times 10^{4}$ is given in Fig. 10 . The core of the flow shows very low azimuthal velocities and indicates an averaged bulk velocity of zero $\left(\bar{U}_{\mathrm{b}} \approx 0\right)$. Substituting $U_{\text {in }}=-U_{\text {out }}$ and $\bar{U}_{\mathrm{b}}=0$ into (11) implies that $C_{f i, 0}=\left(r_{\mathrm{o}} / r_{\mathrm{i}}\right)^{2} C_{f o, 0}$ for the given conditions.

When the friction coefficient is reduced at the inner-cylinder wall due to the riblets, the averaged bulk fluid will start to co-rotate a little with the direction of the outer cylinder. Likewise, when the friction coefficient at the inner wall is increased, the core flow will start to co-rotate a little with the inner cylinder. A similar phenomenon also occurs by operating two smooth cylinder walls with a rotation number $R_{\Omega} \neq 0$. The sign of the rotation number indicates the net system rotation; a negative or positive rotation number is associated with core flow rotation with the direction of the inner- or outer-cylinder rotation, respectively. Hence, a reduced shear stress under exact counter-rotation at the inner-cylinder wall due to riblets will result in a positive apparent rotation number $\hat{R}_{\Omega}$ and vice versa.

Ravelet et al. (2010) demonstrated strong dependency of the friction coefficient $C_{\mathrm{f}}$ to the rotation number $R_{\Omega}$ with the same experimental setup. The friction coefficient $C_{\mathrm{f}}$ has a linear decline around rotation number $R_{\Omega}=0$

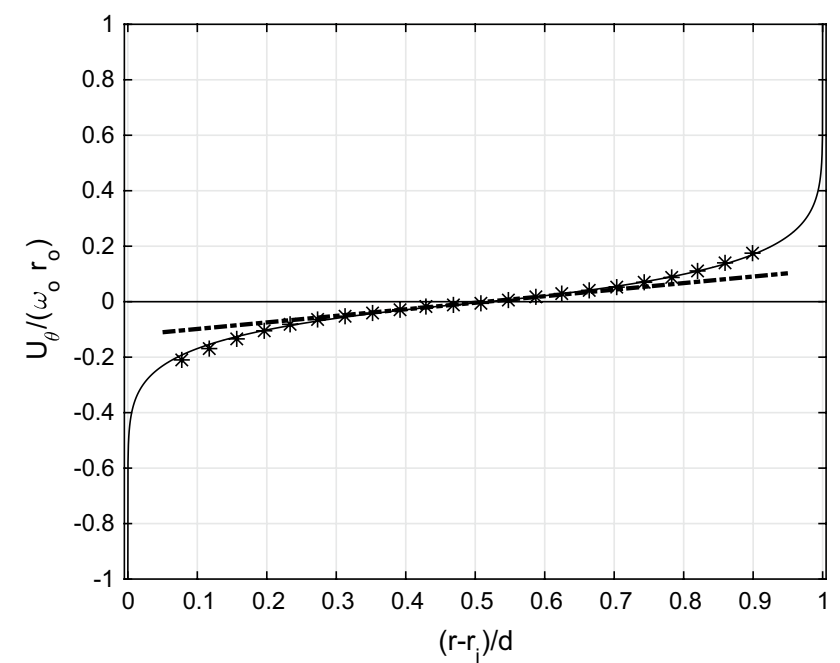

Fig. 10 Averaged azimuthal velocity as a function of the radial position for shear Reynolds numbers $R e_{\mathrm{s}}=4.7 \times 10^{4}$. The velocities are normalized to the azimuthal velocity of the outer cylinder $\left(\omega_{0} r_{0}\right)$. The solid line represents the velocity profile for turbulent boundary layers

with increasing rotation numbers. In the case of riblets, the net system rotation is changed $\left(\hat{R}_{\Omega} \neq 0\right)$ even though we are operating under exact counter-rotating conditions $\left(R_{\Omega}=0\right)$. Except from a riblet drag change, this net system rotation also imposes a change in the friction coefficient.

We propose a simple model to distinguish drag changes due to the rotation effect and the riblet effect by measuring a change in torque on the inner-cylinder wall. We consider the change in friction coefficient of the outer-cylinder wall $\left(C_{f o}\right)$ to be negligible for any Reynolds number $R e_{\mathrm{s}}$, and therefore $C_{f o}=C_{f o, 0}$. The averaged bulk velocity is normalized with the azimuthal velocity of the outer cylinder $U_{\text {out }}$, resulting in $\delta=\bar{U}_{\mathrm{b}} / U_{\text {out }}$.

Equation (11) is rewritten as:

$$
\begin{aligned}
\tau_{w, r i b} & =\frac{1}{2} \rho C_{f, r i b} U_{\text {out }}^{2}(-1-\delta)^{2} \\
& =\frac{1}{2} \rho C_{f o} U_{\text {out }}^{2}(1-\delta)^{2}\left(\frac{r_{\mathrm{o}}}{r_{\mathrm{i}}}\right)^{2} .
\end{aligned}
$$

Rearranging (12) results in:

$C_{f, i, i b}(1+\delta)^{2}=\left(\frac{r_{\mathrm{o}}}{r_{\mathrm{i}}}\right)^{2} C_{f o}(1-\delta)^{2}$.

The comparison between a riblet and smooth inner-cylinder surface can be made, with $C_{f i, 0}=\left(r_{\mathrm{o}} / r_{\mathrm{i}}\right)^{2} C_{f o, 0}=\left(r_{\mathrm{o}} / r_{\mathrm{i}}\right)^{2} C_{f o}$ :

$\frac{\tau_{w, r i b}}{\tau_{w, 0}} \sim \frac{C_{f, r i b}}{C_{f i, 0}}=\frac{C_{f i, r i b}}{\left(r_{\mathrm{o}} / r_{\mathrm{i}}\right)^{2} C_{f o}}=\frac{(1-\delta)^{2}}{(1+\delta)^{2}}$. 
Thus, the change in averaged bulk velocity due to the change in shear stress at the inner-cylinder wall is determined by:

$\delta=\frac{1-\sqrt{\tau_{w, r i b} / \tau_{w, 0}}}{1+\sqrt{\tau_{w, r i b} / \tau_{w, 0}}}$.

According to (15), the maximum drag reduction of $5.3 \%$ observed around Reynolds number $R e_{\mathrm{s}}=4.7 \times 10^{4}$ will correspond with a change in averaged bulk velocity $\delta=0.014$. PIV measurements confirm a similar shift of the averaged bulk velocity when a riblet inner cylinder is used (Fig. 11). The velocity gradient at the inner-cylinder wall decreases significantly by using a riblet inner cylinder, which is associated with a lower level of turbulence. The gradient at the outer-cylinder wall remains unaltered.

The rotation number $R_{\Omega}$ of (2) is equal to:

$R_{\Omega}=(1-\eta) \frac{U_{\mathrm{i}}+U_{\mathrm{o}}}{\eta U_{\mathrm{o}}-U_{\mathrm{i}}}$.

A small shift in the averaged bulk velocity under exact counter-rotating conditions $\left(R_{\Omega}=0\right)$ quantifies the apparent rotation number $\hat{R}_{\Omega}$ :

$\hat{R}_{\Omega} \simeq(1-\eta) \frac{\left(U_{\mathrm{i}}+\bar{U}_{\mathrm{b}}\right)+\left(U_{\mathrm{o}}+\bar{U}_{\mathrm{b}}\right)}{\eta\left(U_{\mathrm{o}}+\bar{U}_{\mathrm{b}}\right)-\left(U_{\mathrm{i}}+\bar{U}_{\mathrm{b}}\right)}$.

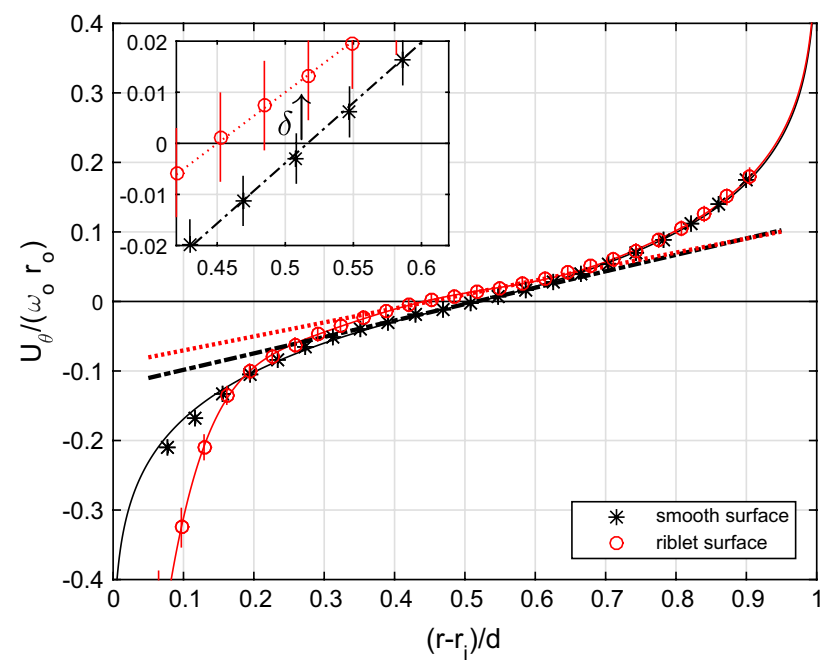

Fig. 11 Azimuthal velocity profiles of a smooth and riblet inner cylinder at Reynolds number $R e_{\mathrm{S}}=4.7 \times 10^{4}$ under exact counterrotating conditions $\left(R_{\Omega}=0\right)$. The velocities are normalized to the azimuthal velocity of the outer cylinder $\left(\omega_{\mathrm{o}} r_{\mathrm{O}}\right)$. Error bars the estimated $95 \%$ confidence interval. The solid lines represent the velocity profile for turbulent boundary layers for each case. The dashed lines indicates the azimuthal core flow velocities, for smooth surface (black dashes) with slope $\alpha \approx 0.25$ and for riblet surface (red dots) with slope $\alpha \approx 0.20$. Inset zero-crossing of the azimuthal velocity profiles
Equation (17) is rewritten as:

$\hat{R}_{\Omega} \simeq(1-\eta) \frac{(-1+\delta)+(1+\delta)}{\eta(1+\delta)-(-1+\delta)}$.

A drag reduction of $5.3 \%$ with an averaged bulk velocity change of $\delta=0.014$ results in an apparent rotation number $\hat{R}_{\Omega}=0.0012$. The apparent rotation number $\hat{R}_{\Omega}$ is very small but sufficient to play a substantial role in the total measured drag change. The relative rotational drag change is the difference between friction coefficients $C_{\mathrm{f}}\left(R_{\Omega}=0\right)$ and $C_{\mathrm{f}}\left(\hat{R}_{\Omega}\right)$ with respect to $C_{\mathrm{f}}\left(R_{\Omega}=0\right)$. The slope $\mathrm{d} C_{\mathrm{f}} / \mathrm{d} R_{\Omega}$ around $R_{\Omega}=0$ defines the magnitude of rotational drag change and is specific for each Reynolds number $R e_{\mathrm{S}}$.

Accurate torque measurements were performed at various rotation numbers $R_{\Omega}$ for Reynolds numbers $R e_{\mathrm{S}}=4 \times 10^{3}$ to $10 \times 10^{4}$. Figure $12 \mathrm{a}$ shows the friction coefficients at a shear Reynolds number $\operatorname{Re}_{\mathrm{s}}=2.0 \times 10^{4}$ for rotation numbers $-2.5 \times 10^{-3}<R_{\Omega}<2.5 \times 10^{-3}$. The slope values $\mathrm{d} C_{\mathrm{f}} / \mathrm{d} R_{\Omega}$ as a function of Reynolds number $R e_{\mathrm{s}}$ are displayed in Fig. 12b. The relative rotational drag change $\left(\Delta \tau_{\theta} / \tau_{w, 0}\right)$ due to the rotation effect for a specific Reynolds number $R e_{s}$ is defined by:

$\frac{\Delta \tau_{\theta}}{\tau_{w, 0}}=\frac{\mathrm{d} C_{\mathrm{f}} / \mathrm{d} R_{\Omega} \cdot \hat{R}_{\Omega}}{C_{\mathrm{f}, 0}}$.

The net drag reduction of riblets is determined by the measured drag change plus the drag change due to the rotation effect. For Reynolds number $R e_{\mathrm{s}}=4.7 \times 10^{4}$ we measured a drag reduction of $5.3 \%$ and found positive rotation of the averaged bulk fluid with an apparent rotation number of $\hat{R}_{\Omega}=0.0012$. Multiplying the slope value $\mathrm{d} C_{\mathrm{d}} / \mathrm{d} R_{\Omega}$ at $R e_{\mathrm{S}}=4.7 \times 10^{4}$ with the apparent rotation number $\hat{R}_{\Omega}$ gives a drag rotation effect of $-1.9 \%$. Consequently, the maximum net riblet drag reduction $\left(\Delta \tau / \tau_{0}\right)_{\max }$ is determined to be $3.4 \%$. The model is applied to all measured drag values in order to determine the actual net drag change by the riblet surface. The results are presented in Fig. 13.

\subsection{Data validation}

The data are compared to other available data of drag reduction research with similar riblets (Bechert et al. 1997, Hall and Joseph 2000) while keeping in mind that riblet performance can be sensitive to experimental geometry, measurement accuracy and riblet surface quality. Figure 14 presents the obtained net drag change $\Delta \tau / \tau_{0}$ as a function of the riblet spacing Reynolds number $s^{+}$measured with the current experimental facility and is limited to $s^{+}>3.5\left(\sim R e_{\mathrm{s}}>10^{4}\right)$.

The overall trend of the curve is comparable with earlier reported results; a maximum drag reduction between riblet 


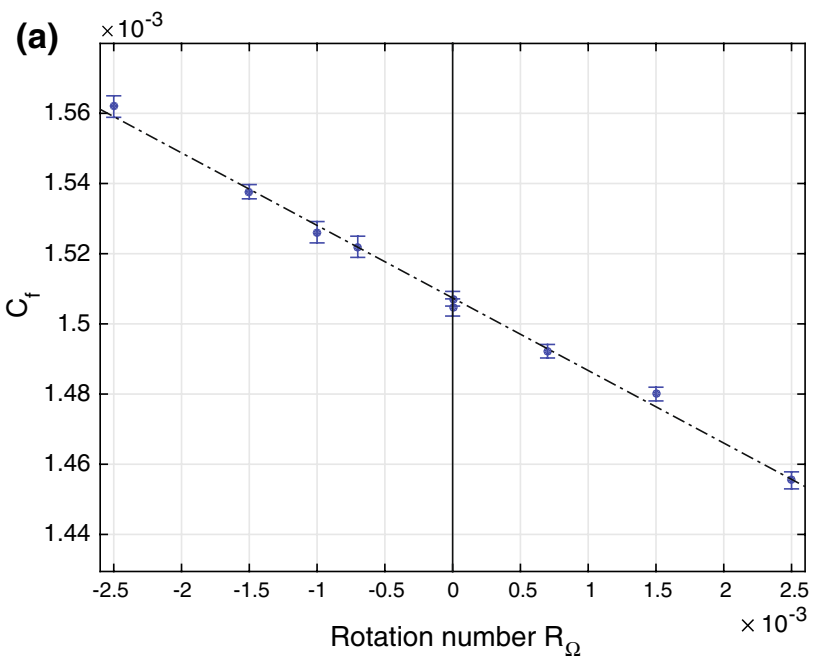

Fig. 12 a Friction coefficient $C_{\mathrm{f}}$ as a function of the rotation number $R_{\Omega}$ at a shear Reynolds number $R e_{\mathrm{s}}=2.0 \times 10^{4}$. The dashed line is the best linear fit of the data points with slope $\mathrm{d} C_{\mathrm{f}} / \mathrm{d} R_{\Omega}=(-20.7 \pm 0.5) \times 10^{-3}$. Error bars $95 \%$ confidence

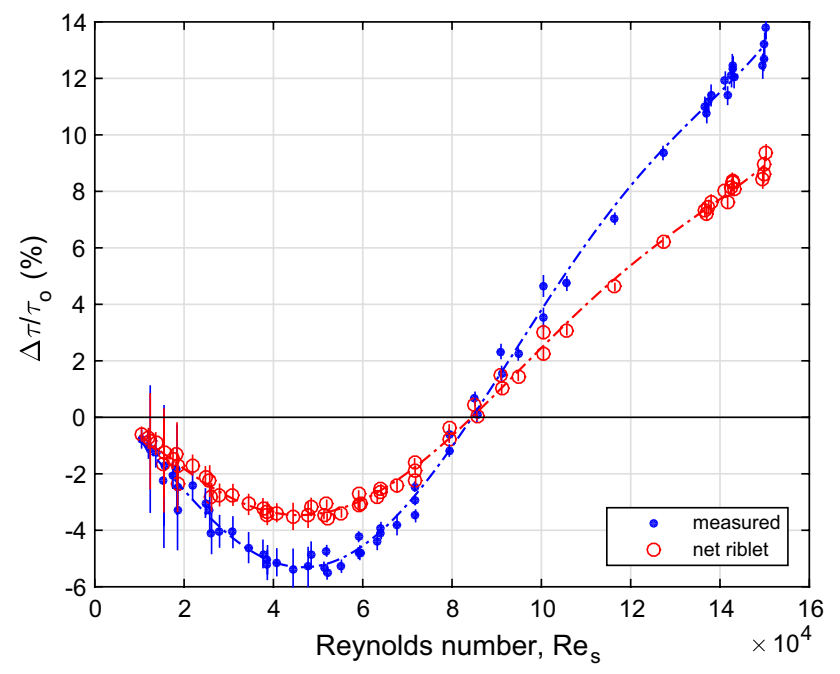

Fig. 13 Measured and net drag change by riblet inner cylinder under exact counter-rotation $\left(R_{\Omega}=0\right)$. The dashed lines are polynomial fits of the obtained results between $R e_{\mathrm{s}}=1.0 \times 10^{4}$ to $15 \times 10^{4}$

spacing $s^{+}=11-16$ and a zero drag reduction cross-over point between $s^{+}=21-27$. Higher riblet spacing $s^{+}$is associated with wall roughness compared to the high skin friction, and the riblets exceed their drag-reducing benefits.

Hall and Joseph (2000) performed similar drag measurements with riblets on the inner-cylinder surface with only outer-cylinder rotation $\left(R e_{\mathrm{i}}=0\right)$ and reported a maximum drag reduction of $5.1 \%$. Their flow is driven by the outer cylinder, while the shear stress is measured on the inner cylinder. No comments are made for global

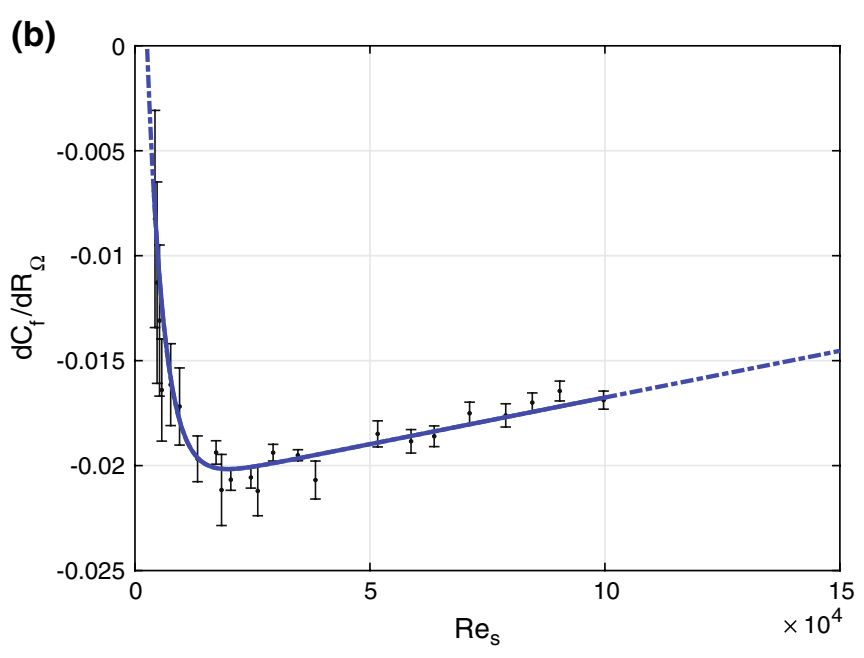

interval. The data at rotation number $R_{\Omega}=0$ is reproducible within $0.17 \%$, b slope values $\mathrm{d} C_{\mathrm{f}} / \mathrm{d} R_{\Omega}$ as a function of Reynolds number $R e_{\mathrm{S}}$ around rotation number $R_{\Omega}=0$. The solid line is a least squares fit of the data, the dashed line is the extrapolation of the solid line

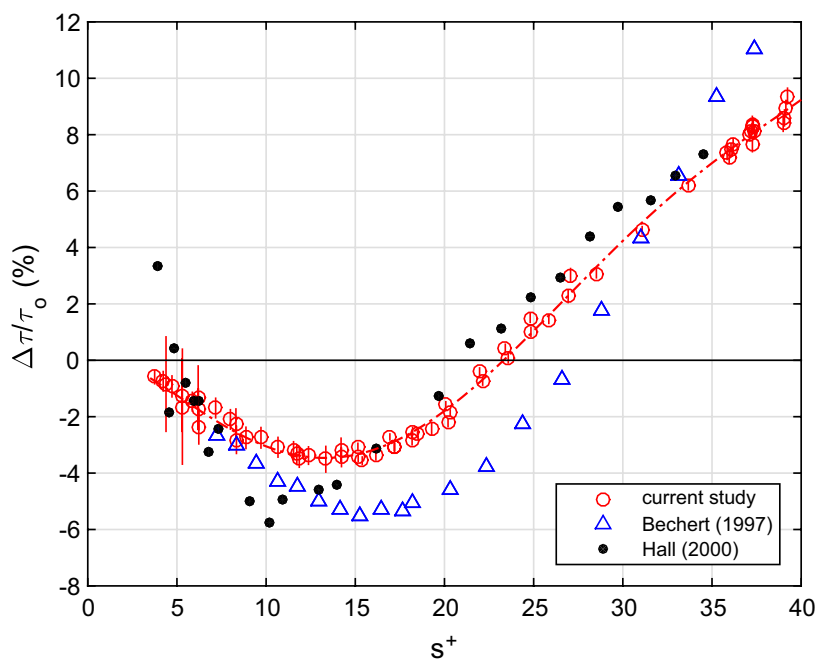

Fig. 14 Net riblet drag change $\Delta \tau / \tau_{0}$ as a function of the riblet spacing $s^{+}$. Compared to drag results obtained in an oil channel (Bechert et al. 1997) and an outer-cylinder rotating Taylor-Couette (Hall and Joseph 2000)

rotation or possible rotation effects, while both phenomena will influence the turbulent structures and velocity fields and consequently manipulate the drag performance of surfaces when compared to boundary layer or fluid channel experiments. Secondly and possibly more important, they observed high-frequency vibrations of the inner cylinder during measurements in the range of $s^{+}=7-20\left(R e_{\mathrm{s}}=1 \times 10^{4}\right.$ to $\left.3 \times 10^{4}\right)$. They reported that their flow is presumably in the midst of a flow transition, which makes direct comparison of the results difficult. 
Bechert et al. (1997) performed their riblet measurements in the Berlin oil channel facility with a very accurate shear stress balance. Their curve looks equivalent to ours, but their observed maximum drag reduction is around $5.5 \%$ at $s^{+}=16$. The zero drag reduction cross-over point is at a higher $s^{+}$-value of $s^{+}=27$, resulting in a wide range of drag reduction. After the cross-over point, the curve has a steeper slope compared to the slope of the current study. The noticeable round/flat area of the riblet tips (Fig. 3) can conceivably be a reason for the deviation in drag results, as the tip sharpness is a crucial factor for the drag-reducing performance of any riblet geometry (Bechert et al. 1997).

\section{Conclusion}

Previous studies have demonstrated that a small amount of drag reduction is achievable by riblets, but requires high accuracy of the experimental setup. The changes in drag can often be within the scatter of the measurements. In our study, the friction coefficients are reproducible within $0.6 \%$ for all working fluids and are more accurate at higher shear Reynolds numbers $R e_{\mathrm{S}}$, where the torque meter is more precise with relative less noise. We verified the presence of different flow regimes by PIV measurements and concluded that the friction coefficients and the drag performance of riblets are strongly determined by the flow regimes. In the Taylorvortex flow regime, the riblets induce extra drag compared to a smooth surface, which is considered to be an artefact of the experimental setup rather than a drag characteristic of riblets. Therefore, the derived riblet data are limited to $R e_{\mathrm{S}}>10^{4}$ for the comparison to the data obtained by other researchers, as the low Reynolds number flow characteristics in a TC facility differ significantly from typical turbulent channel and pipe flows. For fully turbulent flow, the measured torque can be reduced with $5.3 \%$ by applying riblets.

PIV measurements at Reynolds number $R e_{\mathrm{s}}=4.7 \times 10^{4}$ show a change in the azimuthal velocity profile by applying riblets on the inner-cylinder surface. The velocity gradient at the inner-cylinder wall decreases significantly, which is associated with a lower level of turbulence.

A rotation effect occurs by the drag changes under exact counter-rotating conditions. The proposed model indicates the change in mean azimuthal bulk flow velocity. The maximum observed drag reduction of $5.3 \%$ at the inner-cylinder wall implies a change of $1.4 \%$ in the mean azimuthal bulk velocity, which is also verified by PIV measurements. By taking the rotation effect into account, we obtain a proper method to rectify the net drag change by riblets, which reasonably fits earlier reported data.

The Taylor-Couette facility has shown to be a suitable facility for accurate torque measurements to characterize the drag performance of surfaces.
Acknowledgments This research is funded by InnosportNL. Special thanks to Dr.-Ing. Wolfram Hage for providing the riblet data of the Berlin oil channel measurements (Bechert et al. 1997).

Open Access This article is distributed under the terms of the Creative Commons Attribution 4.0 International License (http://creativecommons.org/licenses/by/4.0/), which permits unrestricted use, distribution, and reproduction in any medium, provided you give appropriate credit to the original author(s) and the source, provide a link to the Creative Commons license, and indicate if changes were made.

\section{References}

Andereck CD, Liu S, Swinney HL (1986) Flow regimes in a circular Couette system with independently rotating cylinders. J Fluid Mech 164:155-183

Aydin E, Leutheusser H (1991) Plane-couette flow between smooth and rough walls. Exp Fluids 11(5):302-312

Bechert D, Bruse M, Hage W, Van der Hoeven JT, Hoppe G (1997) Experiments on drag-reducing surfaces and their optimization with an adjustable geometry. J Fluid Mech 338:59-87

Bushnell DM (1990) Viscous drag reduction in boundary layers, vol 123. AIAA, Washington, DC

Daily JW, Nece RE (1960) Chamber dimension effects on induced flow and frictional resistance of enclosed rotating disks. J Fluids Eng 82(1):217-230

Dong S (2008) Turbulent flow between counter-rotating concentric cylinders: a direct numerical simulation study. J Fluid Mech 615:371-399

Dubrulle B, Dauchot O, Daviaud F, Longaretti PY, Richard D, Zahn JP (2005) Stability and turbulent transport in Taylor-Couette flow from analysis of experimental data. Phys Fluids (1994-present) 17(9):095103

Eckhardt B, Grossmann S, Lohse D (2007) Torque scaling in turbulent Taylor-Couette flow between independently rotating cylinders. J Fluid Mech 581:221-250

Elsinga GE, Scarano F, Wieneke B, Van Oudheusden B (2006) Tomographic particle image velocimetry. Exp Fluids 41(6):933-947

Esser A, Grossmann S (1996) Analytic expression for Taylor-Couette stability boundary. Phys Fluids (1994-present) 8(7):1814-1819

Hall T, Joseph D (2000) Rotating cylinder drag balance with application to riblets. Exp Fluids 29(3):215-227

Hudson A, Eibeck P (1991) Torque measurements of corotating disks in an axisymmetric enclosure. J Fluids Eng 113(4):648-653

Kalashnikov V (1998) Dynamical similarity and dimensionless relations for turbulent drag reduction by polymer additives. J NonNewton Fluid Mech 75(2):209-230

Kilic M, Owen JM (2002) Computation of flow between two discs rotating at different speeds. In: ASME Turbo Expo 2002: Power for Land, Sea, and Air, American Society of Mechanical Engineers, 749-759

Koeltzsch K, Qi Y, Brodkey R, Zakin J (2003) Drag reduction using surfactants in a rotating cylinder geometry. Exp Fluids 34(4):515-530

Lathrop DP, Fineberg J, Swinney HL (1992) Transition to shear-driven turbulence in Couette-Taylor flow. Phys Rev A 46(10):6390

Lewis GS, Swinney HL (1999) Velocity structure functions, scaling, and transitions in high-Reynolds-number Couette-Taylor flow. Phys Rev E 59(5):5457

Ravelet F, Delfos R, Westerweel J (2010) Influence of global rotation and Reynolds number on the large-scale features of a turbulent Taylor-Couette flow. Phys of Fluids (1994-present) 22(5):055103 
Taylor GI (1923) Stability of a viscous liquid contained between two rotating cylinders. Philosophical transactions of the royal society of London series A, containing papers of a mathematical or physical character, 289-343

Tokgöz S (2014) Coherent structures in Taylor-Couette flow: Experimental investigation. PhD thesis, TU Delft, Delft University of Technology

Tokgoz S, Elsinga G, Delfos R, Westerweel J (2012) Spatial resolution and dissipation rate estimation in Taylor-Couette flow for tomographic PIV. Exp Fluids 53(3):561-583

Van Bokhoven L, Clercx H, Van Heijst G, Trieling R (2009) Experiments on rapidly rotating turbulent flows. Phys Fluids (1994-present) 21(9):096601 van den Berg TH, Luther S, Lathrop DP, Lohse D (2005) Drag reduction in bubbly Taylor-Couette turbulence. Phys Rev Lett 94(4):044501

van der Hoeven J (2000) Observations in the turbulent boundarylayer-high resolution dpiv measurements over smooth and riblet surfaces. PhD thesis, TU Delft, Delft University of Technology

Watanabe K, Takayama T, Ogata S, Isozaki S (2003) Flow between two coaxial rotating cylinders with a highly water-repellent wall. AIChE J 49(8):1956-1963

Westerweel J, Scarano F (2005) Universal outlier detection for PIV data. Exp Fluids 39(6):1096-1100 